\title{
Development of an efficient perfusion-based protocol for whole-organ decellularization of the ovine uterus as a human-sized model and in vivo application of the bioscaffolds
}

\author{
Seyedeh Sima Daryabari ${ }^{1} \cdot$ Abdol-Mohammad Kajbafzadeh $^{1}$ (D) Kiarad Fendereski $^{1} \cdot$ Fariba Ghorbani $^{1}$. \\ Mehrshad Dehnavi ${ }^{1} \cdot$ Minoo Rostami $^{1} \cdot$ Bahram Azizi Garajegayeh $^{2} \cdot$ Seyed Mohammad Tavangar ${ }^{3}$
}

Received: 23 January 2019 / Accepted: 26 April 2019 / Published online: 16 May 2019

(C) Springer Science+Business Media, LLC, part of Springer Nature 2019

\begin{abstract}
Purpose The main purpose of this investigation was to determine an efficient whole-organ decellularization protocol of a humansized uterus and evaluate the in vivo properties of the bioscaffold.

Methods Twenty-four ovine uteri were included in this investigation and were decellularized by three different protocols ( $n$ 6). We performed histopathological and immunohistochemical evaluations, 4,6-diamidino-2-phenylindole (DAPI) staining, DNA quantification, MTT assay, scanning electron microscopy, biomechanical studies, and CT angiography to characterize the scaffolds. The optimized protocol was determined, and patches were grafted into the uterine horns of eight female Wistar rats. The grafts were extracted after 10 days; the opposite horns were harvested to be evaluated as controls.

Results Protocol III (perfusion with $0.25 \%$ and $0.5 \%$ SDS solution and preservation in $10 \%$ formalin) was determined as the optimized method with efficient removal of the cellular components while preserving the extracellular matrix. Also, the bioscaffolds demonstrated native-like biomechanical, structural, and vascular properties. Histological and immunohistochemical evaluations of the harvested grafts confirmed the biocompatibility and recellularization potential of bioscaffolds. Also, the grafts demonstrated higher positive reaction for CD31 and Ki67 markers compared with the control samples which indicated eminent angiogenesis properties and proliferative capacity of the implanted tissues.

Conclusions This investigation introduces an optimized protocol for whole-organ decellularization of the human-sized uterus with native-like characteristics and a prominent potential for regeneration and angiogenesis which could be employed in in vitro and in vivo studies. To the best of our knowledge, this is the first study to report biomechanical properties and angiographic evaluations of a large animal uterine scaffold.
\end{abstract}

Keywords Uterus $\cdot$ Infertility $\cdot$ Tissue engineering $\cdot$ Regeneration $\cdot$ Bioscaffold

Abdol-Mohammad Kajbafzadeh

kajbafzd@sina.tums.ac.ir

1 Section of Tissue Engineering and Stem Cell Therapy, Pediatric Urology and Regenerative Medicine Research Center, Children's Medical Center, Pediatric Center of Excellence, Tehran University of Medical Sciences, No. 62, Dr. Gharibs Street, Keshavarz Boulevard, Tehran 1419733151, Iran

2 Imaging Center, Children's Medical Center, Tehran University of Medical Sciences, Tehran, Iran

3 Department of Pathology, Shariati Hospital, Tehran University of Medical Sciences, Tehran, Iran

\section{Introduction}

One in every 500 women is suffering from absolute uterine factor infertility (AUFI) which includes women with the inability to reproduce due to the absence of a normal uterus [1]. Several congenital abnormalities or the hysterectomy for various malignant or benign disorders could result in AUFI [2]. Although assisted reproductive techniques are broadly employed in various types of infertilities, these are not applicable for AUFI patients due to the nature of the disorder [3]. Therefore, uterus transplantation is currently the only definitive therapeutic strategy for these patients. The investigations 
by Brännström et al. led to the first live birth from a transplanted uterus in 2015 [4]. Nevertheless, ischemic injuries, mental conditions, etc. for the donor and the adverse outcomes of immunosuppressant therapies of the recipient in allograft transplantation are inevitable [2, 5]. Tissue engineering has the potential to provide a new source of organs to overcome these limitations by autologous recellularization of biological or synthetic scaffolds [5]. In the past recent years, biological scaffolds have gained more interest due to the similarities to the native tissues in biomechanical, functional, and structural properties [6]. The initial efforts in uterine tissue engineering were focused on the production of in vitro models for investigations on malignant cells' invasion [7]. Furthermore, several studies evaluated the efficacy of engineered patches for uterine reconstruction [8]. The application of acellular uterine tissue as a bioscaffold for tissue regeneration has resulted in prosperous outcomes. The elimination of antigen-presenting cells during the decellularization process prevents any immunological reactions against the grafts. Also, the preserved extracellular matrix (ECM) comprises vital structural molecules in addition to growth factors which stimulate cellular migration, proliferation, and differentiation [6]. In this regard, investigations by Santoso et al. in 2014 and Hiroka et al. in 2016 provided proof of concept for tissue regeneration by application of acellular uterine tissue segments. The animals in both studies were able to reproduce following the implantation $[9,10]$.

Recently, whole-organ decellularization has been proposed as a technique to provide scaffolds with intact vascular network and a three-dimensional structure similar to the native tissue. These substantial features are advantageous for organ recellularization through the vascular conduits. Also, preservation of the organ structure provides the required biomechanical properties for organ regeneration which is particularly essential in the uterus due to its expansion during the pregnancy [11]. Several protocols hitherto have been developed for rat uterus decellularization. Miyazaki and Maruyama investigated the uterine decellularization by sodium dodecyl sulfate (SDS) and Triton X-100, as well as subsequent in vitro recellularization of the scaffold with uterine cells and mesenchymal stem cells [12]. Moreover, in a study by Hellström et al., three protocols were compared regarding decellularization efficacy and preservation of the ECM in rat uterus [11]. A subsequent study by Hellström et al., as well as the one by Miyazaki and Maruyama, included implantation of recellularized uterine patches, and occurrence of normal pregnancies confirmed the preservation of the reproductive potential in the recipient rats in both studies $[12,13]$. Although these studies provided a basis for further application of tissue engineering techniques, several steps are required to employ this therapeutic strategy in human models. A major challenge is to develop efficient whole-organ decellularization protocols in large animal uteri. In this regard, Campo et al. described the first successful decellularization of pig uterus as a large animal model using the detergent agents with and without a heat shock [14].

In the present study, we successfully decellularized the whole ovine uteri by three different perfusion-based protocols. We evaluated the efficacy of each method for tissue decellularization as well as the preservation of the ECM structure and components. The bioscaffolds' biomechanical properties were characterized; the vascular network maintenance was assessed, and biocompatibility or toxicity of the scaffolds and removal of the detergent agents were studied. Subsequently, we determined the optimized decellularization protocol of the ovine uterus and implanted $10 \times 5 \times 5 \mathrm{~mm}^{3}$ segments of the bioscaffolds into the uterine horns of rat models to investigate the in vivo characteristics of the biomaterial. Finally, the grafts were extracted after 10 days, and histological and immunohistochemical evaluations were carried out to assess the possible recellularization process as well as the biocompatibility of the scaffolds.

\section{Materials and methods}

All the animal procedures were approved by The Animal Ethics Committee of Tehran University of Medical Sciences and were performed in accordance with the Animal Welfare Act and the Guide for the Care and Use of Laboratory Animals.

\section{Decellularization of the ovine uterus}

We obtained 24 adult and fertile sheep uteri (mean body weight $40 \mathrm{~kg}$ ) regardless of their reproductive status from a local slaughterhouse. The organs were kept in phosphatebuffered saline (PBS; Life Technologies, Carlsbad, CA) solution at $4{ }^{\circ} \mathrm{C}$ and were immediately transferred to the laboratory. Six uteri were kept in a PBS solution at $4{ }^{\circ} \mathrm{C}$ for further evaluations as control models. As for the others, we stripped the organs of the connective tissue, cannulated uterine arteries by $20-\mathrm{G}$ angiocatheters, and fixed the cannulas by $4-0$ silk ligatures. Subsequently, we connected the uterus to a peristaltic pump (CESCO Bellofeeder 1300, CESCO Bioengineering, Taichung, Taiwan) and set the flow rate at $10 \mathrm{ml} / \mathrm{min}$ for each artery. All the procedures were performed at room temperature unless mentioned otherwise. The uteri were decellularized by three different protocols. We used distilled water as the diluent for all solutions. Six uteri were perfused with PBS for $6 \mathrm{~h}$ to evacuate the remaining blood, followed by $1 \%$ SDS (Merck, Darmstadt, Germany) perfusion for $48 \mathrm{~h}, 1 \%$ Triton X-100 (Merck, Darmstadt, Germany) for $24 \mathrm{~h}$, and $72 \mathrm{~h}$ of PBS to remove the remaining detergents (protocol I/P1). Six uteri were decellularized by the second protocol which was extracted from Hellström et al. method of rat uterus decellularization [13]. This protocol consisted of 
perfusion with PBS for 6 h, $4 \%$ DMSO for 48 h, $1 \%$ Triton X100 for $16 \mathrm{~h}$, and final PBS rinsing for $72 \mathrm{~h}$ (protocol II/P2). Furthermore, six uteri were decellularized by a modified method of porcine liver decellularization [15]. Previous studies have also employed liver decellularization protocols for uterine tissue due to certain similarities of the tissue's characteristics [12]. The uteri were perfused by distilled water for $24 \mathrm{~h}, 0.25 \%$ SDS for $24 \mathrm{~h}$, followed by $48 \mathrm{~h}$ of preservation in $0.25 \%$ SDS solution at $4{ }^{\circ} \mathrm{C}$ on a mechanical shaker regulated at 60 RPM. Subsequently, the organs were perfused with $0.5 \%$ SDS for $24 \mathrm{~h}$, followed by distilled water perfusion for $24 \mathrm{~h}$, and $4 \mathrm{~h}$ of preservation in $10 \%$ neutral buffered formalin (Merck, Darmstadt, Germany) at room temperature for collagen cross-linking. Finally, the organs were washed by PBS perfusion for $96 \mathrm{~h}$ to remove the chemical agents (protocol III/P3). We briefly summarized the steps to each protocol in Table 1.

\section{Histological and immunohistochemical evaluations of the scaffolds}

Following the decellularization process, we obtained $1-\mathrm{cm}^{3}$ segments from the middle portion of the native and acellular uterine horn tissues for further evaluations and immediately fixed the specimens in 10\% neutral buffered formalin (Merck, Darmstadt, Germany) overnight to prevent any degeneration or distortion of proteins. Subsequently, the samples were dehydrated by graded series of ethanol, were paraffin embedded, sectioned into 5- $\mu \mathrm{m}$ slices, and stained with hematoxylin and eosin (H\&E), Masson's trichrome, Picrosirius red, and Alcian blue to evaluate the ECM content of the scaffolds. Masson's trichrome staining was employed to discriminate between muscle, collagen fibers, and fibrin. Alcian blue staining was performed to determine the ECM content of glycosaminoglycans (GAGs). Picrosirius red staining was carried out to determine the collagen fibers [type I collagen: yelloworange birefringence; type III collagen: green birefringence], and the specimens were visualized under polarized light. Furthermore, immunohistochemical assessments were carried out for specific ECM components including type I collagen (1:100, ab34710, Abcam, Cambridge, UK), type IV Collagen (1:100, ab6586, Abcam, Cambridge, UK), elastin (1:100, ab21610, Abcam, Cambridge, UK), fibronectin (1:100, ab23751, Abcam, Cambridge, UK), and laminin (1:100, ab11575, Abcam, Cambridge, UK) by specific antibodies.

Finally, to obtain quantitative data of positive-stained samples in all groups and verify the preservation of ECM integrity, IHC slides undergone image analysis (pixel/ $/ \mathrm{m}^{2}$ ) by ImageJ software (Wayne Rasband Analytics, National Institutes of Health, USA). Statistical analysis was performed to compare the obtained quantitative data, and a $P$ value of less than 0.05 was considered as the level of significance.

\section{DAPI staining and DNA quantification}

We evaluated the efficacy of the decellularization process by 4,6-diamidino-2-phenylindole (DAPI) staining of the remaining nuclear DNA. We obtained 5- $\mu$ m sections of paraffinembedded samples, deparaffinized them, incubated the specimens in $1 \mu \mathrm{g} / \mathrm{ml}$ DAPI solution (Sigma, St. Louis, MO, USA) for $15 \mathrm{~min}$, and rinsed them with PBS for another $15 \mathrm{~min}$. A fluorescence microscope with a UV filter was employed to visualize DAPI bound to A-T-rich regions of dsDNA. The absence of nuclear materials was considered as complete decellularization of the samples. To perform the quantification test, the total DNA content was extracted from each sample by a genomic DNA purification kit (Thermo Scientific, Lithuania), and the concentration was determined by NanoDrop spectrophotometry (Thermo Scientific NanoDrop 1000) and agarose gel. Finally, we normalized the data to the weight of each sample and compared between the findings.

\section{MTT assay}

The scaffolds' cytotoxicity and biocompatibility were evaluated by 3-[4,5-dimethylthiazol-2-yl]-2,5-diphenyltetrazolium bromide (MTT) assay. This test determines the cell viability rate based on yellow tetrazolium salt MTT conversion to purple formazan crystals in the living cells' mitochondria by measuring the absorbance level of the samples using a spectrophotometer. We employed rat mesenchymal stem cells (MSCs) to evaluate cells' viability and to determine the efficacy of toxic detergents' removal from the scaffolds for each protocol. The
Table 1 The steps to each decellularization protocol. The uteri were decellularized by three different protocols. The peristaltic pump flow rate was set at $10 \mathrm{ml} /$ min for each artery

\begin{tabular}{|c|c|c|}
\hline Protocol I (P1) & Protocol II (P2) & Protocol III (P3) \\
\hline $\begin{array}{l}\cdot \text { PBS perfusion for } 6 \mathrm{~h} \\
\cdot 1 \% \text { SDS perfusion for } 48 \mathrm{~h} \\
\text { - } 1 \% \text { Triton X-100 for } 24 \mathrm{~h} \\
\text { - PBS perfusion for } 72 \mathrm{~h}\end{array}$ & $\begin{array}{l}\text { - PBS perfusion for } 6 \mathrm{~h} \\
\text { - } 4 \% \text { DMSO for } 48 \mathrm{~h} \\
\text { - } 1 \% \text { Triton X-100 for } 16 \mathrm{~h} \\
\text { - PBS perfusion for } 72 \mathrm{~h}\end{array}$ & $\begin{array}{l}\text { - Distilled water perfusion for } 24 \mathrm{~h} \\
\text { - Preservation in } 0.25 \% \text { SDS on shaker } \\
\text { plate for } 48 \mathrm{~h} \\
\text { - } 0.5 \% \text { SDS perfusion for } 24 \mathrm{~h} \\
\text { - Distilled water perfusion for } 24 \mathrm{~h} \\
\text { - Preservation in } 10 \% \text { formalin for } 4 \mathrm{~h} \\
\text { - PBS perfusion for } 96 \mathrm{~h}\end{array}$ \\
\hline
\end{tabular}


test was performed as a previously described protocol with few modifications [16]. Briefly, the MSCs were cultured in Dulbecco's modified Eagle's medium (DMEM) containing $10 \%$ fetal bovine serum at $37^{\circ} \mathrm{C}$ in a $\mathrm{CO}_{2}$ incubator for 1 week. Subsequently, the cells were placed in a 96-well plate, and the bioscaffolds were added; they were incubated for $72 \mathrm{~h}$ at $37^{\circ} \mathrm{C}$. Finally, we added $100 \mu \mathrm{l}$ of MTT solution $(0.5 \mathrm{mg} / \mathrm{ml}$ in PBS) to each well. Following $6 \mathrm{~h}$ of incubation at $37^{\circ} \mathrm{C}$, a multi-well microplate reader (ICN, Switzerland) was employed to determine the optical density at $545 \mathrm{~nm}$; the results were normalized to the control optical density, and survival rates were calculated for each group using the following formula. The tests were carried out three times for each group.

Toxity $\%=\left(1-\frac{\text { mean OD of sample }}{\text { mean OD of control }}\right) \times 100$

Viability $\%=100-$ toxicity $\%$

\section{Scanning electron microscopy}

We performed SEM evaluations to determine the efficacy of the protocols for decellularization and preservation of the ECM structure. We provided $1-\mathrm{cm}^{3}$ specimens from the body and horns of native and acellular uteri. The samples were kept in $2.5 \%$ glutaraldehyde at $4{ }^{\circ} \mathrm{C}$ for $45 \mathrm{~min}$, washed with distilled water for three times, and were dehydrated by graded series of ethanol and preservation under a laminar hood for $24 \mathrm{~h}$. The samples were gold sputtered and visualized by SEM (Vega, TESCAN, Brno, Czech Republic) at 30-kV voltage.

\section{Biomechanical characterization}

The biomechanical properties of the native tissue and acellular scaffolds were assessed with a tensile test by a dynamic servohydraulic testing machine (Zwick/Roell, Model: Hct $25-400$, Germany). We provided $8 \times 2 \mathrm{~cm}^{2}$ segments from the uterus horns for this test and attached them to the sample holders. Subsequently, the samples were subjected to $1-\mathrm{kN}$ calibrated load cell with a rate of $10 \mathrm{~mm} / \mathrm{min}$ at room temperature until the force load vanished and tissue rupture was detected by the device. The tests were performed with three replicates for each sample ( $n 3$ ), and force and displacement data were recorded. Finally, we calculated the strain by dividing the displacement by initial gauge length and analyzed the data by SPSS software using ANOVA test. The results were expressed as mean $\pm \mathrm{SE}$, and a $P$ value of less than 0.05 was considered as the significance level.

\section{Computer tomography angiography}

Computer tomography (CT) angiograms were provided to investigate the vascular pattern of the scaffolds and to compare the impact of the decellularization processes on the vascular network and microvascular structure. In this regard, $20 \mathrm{ml}$ of $50 \%$ iodixanol (GE Healthcare, Cork, Ireland) was manually injected through the uterine arteries, and $\mathrm{CT}$ angiography (BrightSpeed 16, Tampa, FL) was carried out in the arterial phase at $10 \mathrm{kV}$ and $50 \mathrm{~mA}$. Finally, the images were reconstructed by a multi-segment algorithm, and a doubleblinded expert in the field of CT angiographic studies analyzed both original and reconstructed images.

\section{Bioscaffold implantation and evaluation of the recellularization process}

Following the evaluation and characterization of the acellular tissues, protocol III samples demonstrated the highest amount of ECM content preservation, the highest level of biocompatibility and cell viability, and the most affluent vascular network while adequate removal of the nuclear components. Thus, protocol III was determined as the optimal decellularization method, and the obtained bioscaffolds were employed for further in vivo investigation. We obtained eight $10 \times 5 \times 5 \mathrm{~mm}^{3}$ segments of P3 scaffolds and implanted them into the right uterine horns of eight adult fertile female Wistar rats (200-250 g of body weight) to investigate the in vivo recellularization process as well as the biocompatibility of these scaffolds. The animals were anesthetized by intramuscular injection of ketamine $(80 \mathrm{mg} / \mathrm{kg})$ and xylazine $(10 \mathrm{mg} / \mathrm{kg})$; the lower abdominal area was shaved, and the rats were placed in the supine position. Subsequently, a midline abdominal incision was made to expose the bladder which we gently shifted to the left side and covered it by moist gauze to avoid dehydration. Following the translocation, the rat uterus was detected posterior to the bladder. The right uterine horn was clamped using a microforceps; we made a $10-\mathrm{mm}$ longitudinal incision on the right uterine horn and implanted the bioscaffold patches using interrupted 5-0 nylon sutures. Finally, the muscular layer and the skin were sutured in double layers by continuous 5-0 Prolene stitches (Fig. 6). After 10 days of follow-up, the grafts were harvested and fixed in 10\% $(w / v)$ neutral buffered formalin (Merck, Darmstadt, Germany) for further histological and immunohistochemical assessments. Also, the opposite uterine horns of the rats were extracted by electrocauterization and were fixed in $10 \%$ formalin for further evaluations. The specimens were histologically studied by H\&E, Alcian blue, and Masson's trichrome staining. Also, immunohistochemical evaluations were performed using antibodies for cytokeratin (MAD-211000Q, Master Diagnostica, Spain), alfa-smooth muscle actin ( $\alpha$-SMA; M: 0851, Dako, Denmark), CD31 (M: 0823, Dako, Denmark), Ki-67 (MAD-020310Q Master Diagnostica, Spain), and vimentin (M: 7020, Dako, Denmark). 


\section{Statistical analysis}

Statistical analysis was carried out by independent sample $t$ test of SPSS, V19 (IBM, SPSS Statistics, IBM Corporation. Chicago, IL, USA). Tensile strength data was analyzed using ANOVA. The results were expressed as mean $\pm \mathrm{SE}$. The level of significance was considered as $p$ value $<0.05$.

\section{Results}

\section{Macroscopic, histological, and immunohistochemical evaluation of the bioscaffolds}

\section{Macroscopic assessment}

Following the decellularization process, all of the samples became transparent which was considered as a sign of decellularization. The macroscopic structure of the acellular organs could not be distinguished from the native ones (Fig. 1).

\section{Histological staining of the bioscaffolds}

Histological evaluation of the acellular scaffolds by H\&E staining demonstrated the absence of nuclear components indicative of complete decellularization in all three groups. These findings were confirmed by DAPI staining and DNA quantification. The endometrial and myometrial ECM structures were maintained, and no considerable distortion could be detected following the decellularization process. Also, vascular conduits were detectable in all of the samples which could be considered as the preservation of the microvascular network. Overall, H\&E staining showed no prominent dissimilarity between the protocols. Masson's trichrome staining of P2 and P3 specimens showed an abundance of collagen fibers and were similar to the native tissue indicating the preservation of the ECM after the decellularization; however, P1 samples showed slightly reduced bundles. In contrast to the native samples, the absence of smooth muscle cells was observed in all acellular scaffolds. Also, preservation of the vessel was a notable finding in all three groups. Picrosirius red staining was indicative of preservation of collagen and elastin fibers in all

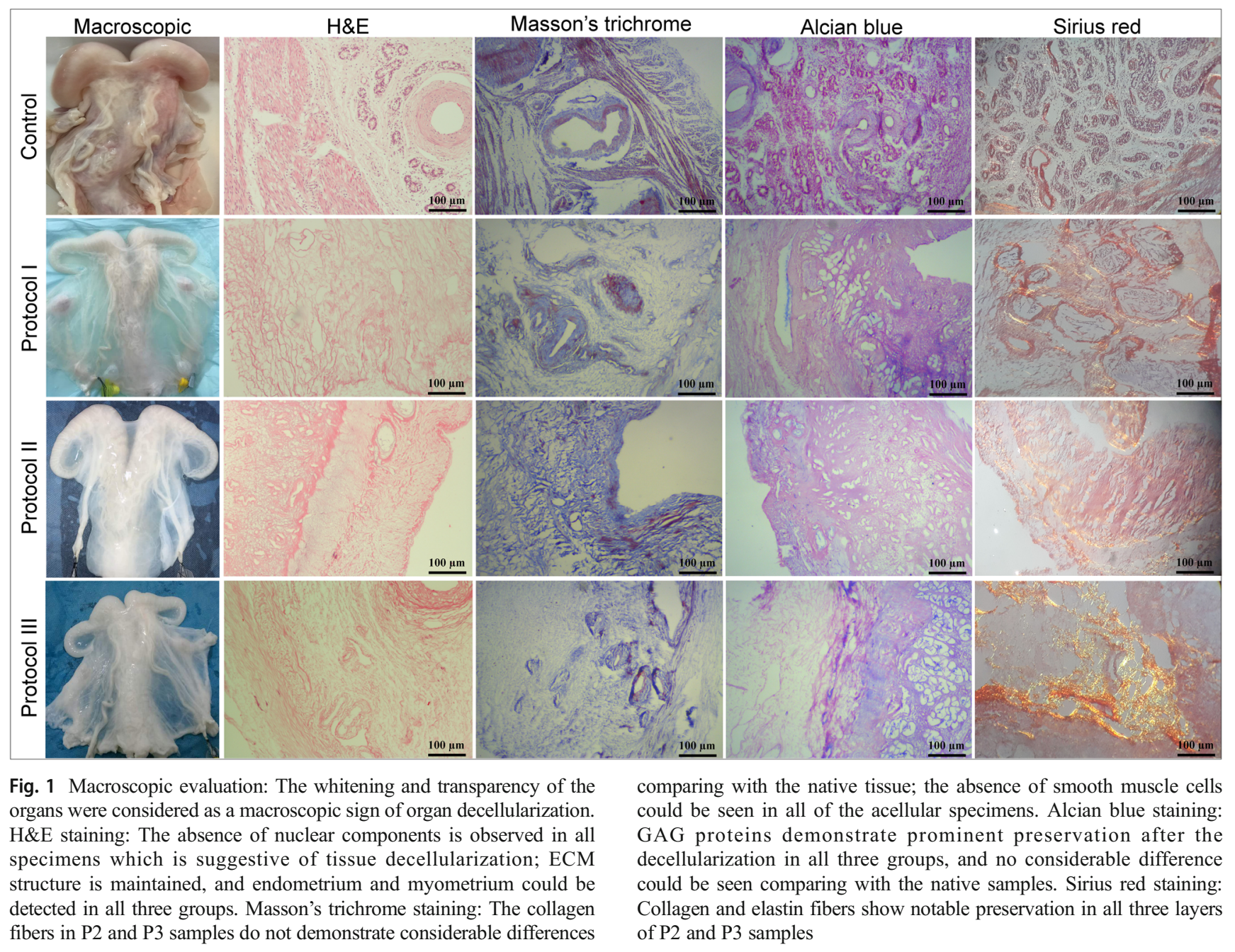


layers of P2 and P3 samples, while P1 specimens showed a considerable loss of these fibers after the decellularization compared with the native uterus. Alcian blue staining of the specimens did not demonstrate any notable difference between the three protocols and the native samples, and the findings were suggestive for the preservation of GAG proteins after the decellularization in all three groups (Fig. 1).

\section{Immunohistochemical staining and analysis}

Immunohistochemical staining and the quantitative data provided by image analysis of the samples showed a high concentration of type I collagen in all study groups; however, type IV collagen was considerably reduced in P1 specimens. Also, elastin fibers did not show any significant decrease in P2 and P3 compared with the native group, while P1 samples demonstrated an immense loss of elastin fibers. IHC assessment of the acellular scaffolds showed that the concentration of fibronectin proteins was prominently decreased in P1 and P3 samples. Laminin fibers in P3 were statistically similar to the native specimens, while $\mathrm{P} 1$ and $\mathrm{P} 3$ depicted a mild reduction $(p<0.05)$. Overall, $\mathrm{P} 2$ and P3 decellularization methods resulted in prominently preserved tissue structure and ECM components in the histological and immunohistochemical evaluation (Fig. 2).

\section{DAPI staining and DNA quantification}

The decellularization of the uteri was confirmed by both DAPI staining and DNA quantification, and the data were compared with samples of the native tissue. In comparison, P1 was the most efficient method for removal of cells and nuclear materials. In contrast, P2 samples showed the highest amount of cellular remnants after the decellularization process in comparison with $\mathrm{P} 1$ and $\mathrm{P} 3(p<0.05)$. This data is presented in Fig. 3.

\section{MTT assay}

The cell-scaffold biocompatibility is essential for further in vitro and in vivo applications of acellular tissues. The biocompatibility of the scaffolds was determined by MTT assay, and each groups' data were compared with a scaffold-free control group. The cell viability was higher than $80 \%$ in all of the samples; however, the lowest viability rate was detected in P2 (83\%). P1 and P2 samples demonstrated a significant decrease in cell viability comparing with the control samples which is probably due to detergent agents' residuals $(p<0.05)$. Based on the data summarized in Fig. 3, P3 demonstrated the highest level of cell viability and showed no significant difference compared with the control group $(p<0.05)$ (Fig. 3).

\section{SEM}

Scanning electron microscopy evaluations demonstrated cobblestone-shaped elements in the native tissue which were considered as cellular components. The replacement of these elements with empty pores in acellular samples was indicative of complete decellularization in all protocols. Also, acellular samples showed no malformation or distortion comparing with the native tissue. The samples provided from different areas of the uterus did not demonstrate any notable divergence and could not be discerned. These findings could be interpreted as the preservation of the ECM ultra-structure following the decellularization process. Altogether, SEM assessments indicated the decellularization of the uteri as well as the preservation of the ECM structure, and no considerable difference was detectable between the three protocols' specimens (Fig. 4).

\section{Biomechanical evaluations}

The ultimate tensile strength, elastic modulus, and strain at failure for each sample were calculated from stress-strain curves. Figure 5 shows the results for the tensile strain and stress of elasticity. Native tissue's Young's modulus was $0.8 \mathrm{MPa}$ that was significantly lower than that of protocol 1 (4.5 MPa) and 2 (3.2 MPa) $(p<0.05)$. These findings could be due to imperative loss of elastin and fibronectin fibers demonstrated by IHC staining in P1 and P2 samples.

In comparison, Young's modulus of protocol III (1.63 MPa) was not significantly different from the native tissue, and P3 samples showed the highest rate of elasticity to the tensile force. The importance of these findings is related to the physiological role of the uterine tissue and the requirement for expansion during the pregnancy time, and an optimal substitute of the natural uterus should contain biomechanical characteristic similar to the native tissue (Fig. 5).

\section{CT angiography}

Evaluation of the reconstructed CT angiography images depicted intact uterine large vessels in all specimens. The acellular tissues in P1 and P2 groups demonstrated considerably preserved vascular network in comparison with the native organ; however, P3 samples showed the most affluent microvasculature between the three protocols. The uterine arteries and its branches were prominently maintained in P3 specimens. Overall, P3 displayed the highest capability of conduits' preservation (Fig. 5).

\section{Bioscaffold implantation and the recellularization process}

The animals were healthy after 10 days, and all of them survived through the follow-up period; the implanted ovine 


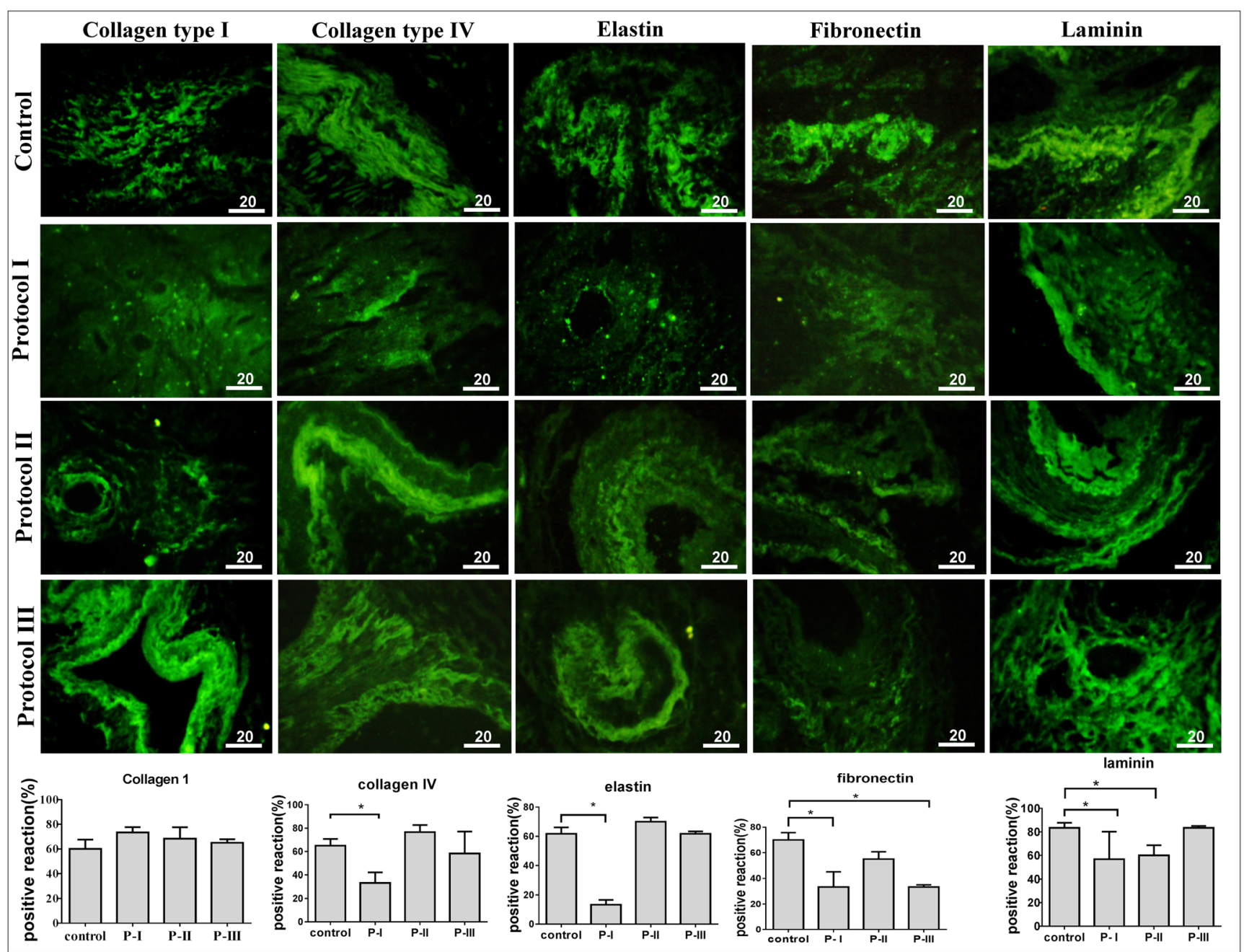

Fig. 2 Immunohistochemical staining and analysis of the samples showed higher than $60 \%$ positive reaction for type I collagen in all study groups, and statistical analyses showed no significant difference between the acellular samples and the native tissues $(p>0.05)$. A considerable loss of type IV collagen was observed in protocol I specimens (approximately 30\% positive reaction for type IV collagen). The other two study groups demonstrated at least $60 \%$ positive reaction for type IV collagen, and the difference was not significant comparing with the native samples $(p>0.05)$. Elastin fibers did not show any significant decrease in protocol II and protocol III compared with the native samples, and positive staining was observed in higher than $60 \%$ of surface. Nevertheless, protocol I revealed a significant decrease (nearly
$10 \%$ positive reaction) in elastin fibers compared with the native tissue protocol I. The concentration of fibronectin proteins was significantly decreased in protocol I and protocol III samples (nearly 10\% positive reaction). On the contrary, protocol II specimens showed prominent preservation of fibronectin proteins, and the statistical evaluation was indicative for no significant difference comparing with the cellular tissue. Laminin fibers were highly maintained in protocol III samples (80\% positive reaction) which was similar to the control group, while protocol I and protocol II depicted a considerable reduction in comparison with the native samples $(p<0.05)$. P-I protocol I, P-II protocol II, P-III protocol III. Asterisk means significant difference $(p<0.05)$ scaffolds were harvested after 10 days and were examined by histological and immunohistochemical techniques, and the results were compared with normal rat uterine tissue.

\section{Histological staining of the harvested scaffolds}

Histological evaluation of the grafted acellular tissues demonstrated a fibro-connective tissue with rich vasculature and thin endometrial layer. H\&E and Alcian blue staining revealed the endothelial layer as the inner epithelial layer in both implant and control samples. Moreover, collagen fibers surrounded by an abundance of smooth muscle fibers were observed in the myometrium of both groups. Trichrome staining illustrated collagen fibers as blue fibers in all of the specimens. Tissue layers and components did not demonstrate any considerable difference between the two groups; endometrium and myometrium could be detected in the grafted and the normal tissue, and these findings were confirmed by immunohistochemical analyses. Also, we were not able to distinguish any sign of inflammatory cell infiltration throughout the specimens in high power field histological evaluations. Furthermore, the increased number of small- to medium- 


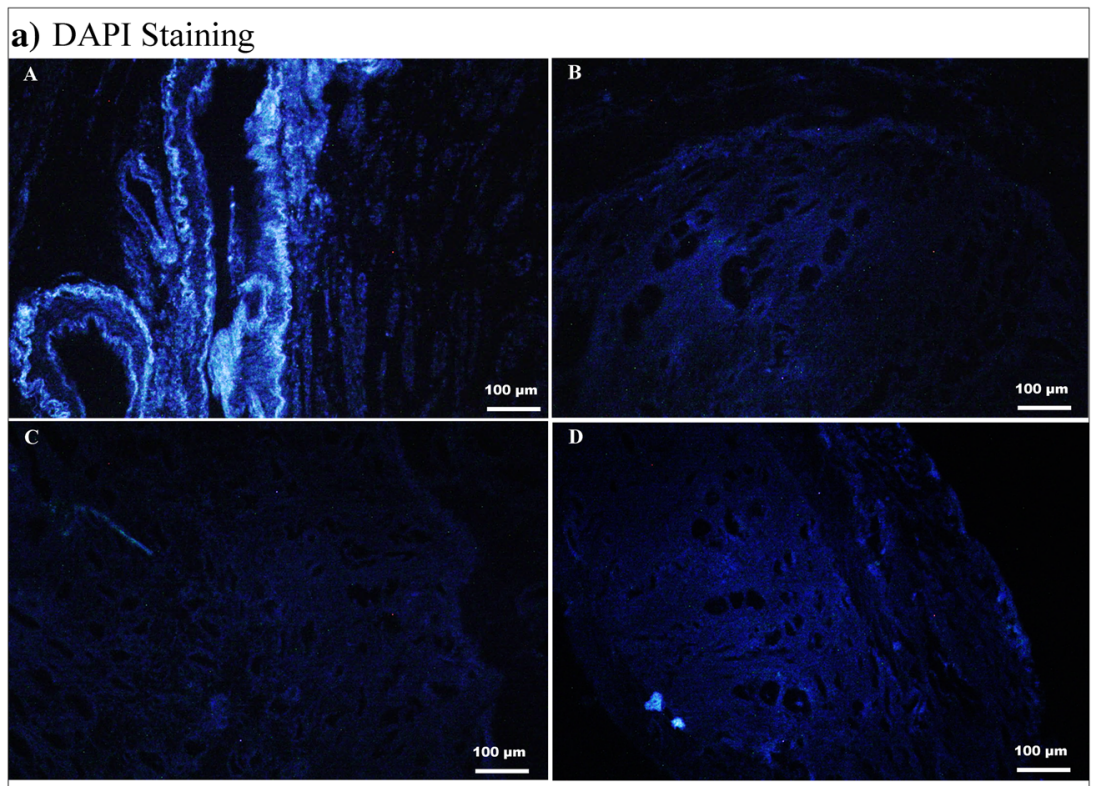

b) DNA quantification

\begin{tabular}{|l|c|}
\hline \multicolumn{1}{|c|}{ Sample } & ng of DNA / $\mathbf{m g}$ of dry tissue \\
\hline Native tissue & $180 \pm 64$ \\
\hline Protocol I & $24.3 \pm 6.3$ \\
\hline Protocol II & $54.3 \pm 21.7$ \\
\hline Protocol III & $44.7 \pm 10.3$ \\
\hline
\end{tabular}

Fig. 3 DAPI (a) staining and DNA quantification (b) of the native tissue (A) and acellular samples (middle portion of the uterine horns) suggested that protocol I (B) was the most efficient method for nuclear component removal with $24.3 \pm 6.3 \mathrm{ng}$ of DNA/mg of dry tissue. Protocol II (C) samples showed the highest amount of cellular remnants with $54.3 \pm$ $21.7 \mathrm{ng} / \mathrm{mg}$ of DNA after the decellularization process in comparison with protocol I and protocol III (D). DNA measurement of protocol III demonstrated $44.7 \pm 10.3 \mathrm{ng}$ of DNA $/ \mathrm{mg}$ of dry tissue which was significantly decreased compared with the native samples $(p<0.05)$. MTT

sized vessels in the implanted tissues comparing with the controls was suggestive of an angiogenesis process which was subsequently confirmed by the IHC staining for CD31 endothelial marker.

\section{Immunohistochemical evaluation of the recellularized samples}

Immunohistochemical evaluation of the samples for cytokeratin marker revealed the endometrium as the inner epithelial layer in native and grafted tissues. Alfa-SMA staining showed a considerable positive reaction in both groups which confirmed the presence of smooth muscle cells in the myometrium of the native and grafted specimens. IHC examination for CD31 marker, as an indicator of the endothelial layer, to investigate the angiogenesis in the implanted scaffolds showed diffused positive reaction in both groups. c) MTT assay

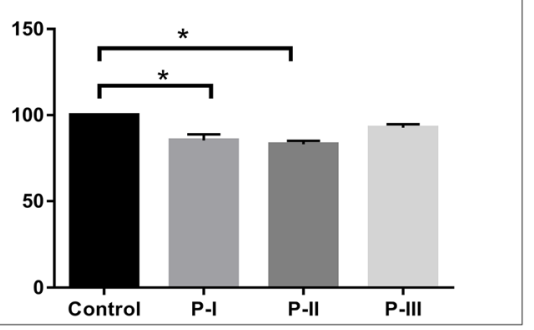

assay (c) shows cell viability of higher than $80 \%$ in all of the bioscaffolds; however, the lowest viability rate is detected in protocol II specimens $(83 \%)$, which is significantly lower than the control samples $(p<0.05)$. Protocol III demonstrates the highest level of cell viability with no significant difference comparing with the native samples $(p>0.05)$. Protocol I scaffolds demonstrate more than $85 \%$ of cell viability following the decellularization process which is significantly lower than the control samples $(p<0.05)$. P-I protocol I, P-II protocol II, P-III protocol III. Asterisk means significantly difference $(p<0.05)$

Nevertheless, the grafted samples demonstrated increased CD31-positive cells which are suggestive of an angiogenesis process within the recellularized tissue. We performed IHC staining for Ki-67 to determine the proliferative capacity of the grafts. Native uterine tissue showed a weak positive reaction for Ki-67 marker with a higher concentration of positivestained cells in the endometrium which is probably due to endometrial shedding and typical characteristics of this layer. On the contrary, the implanted samples demonstrated a prominently higher positive reaction for Ki-67 marker both in the endometrium and the myometrium of the tissues which could be interpreted as the high proliferative capacity of the grafted tissue and confirms the presence of a regenerative process in the acellular tissue. IHC staining for vimentin illustrated the fibro-connective tissue in the myometrium of both samples, and no prominent dissimilarity could be detected between the two groups. Overall, histological and immunohistochemical 


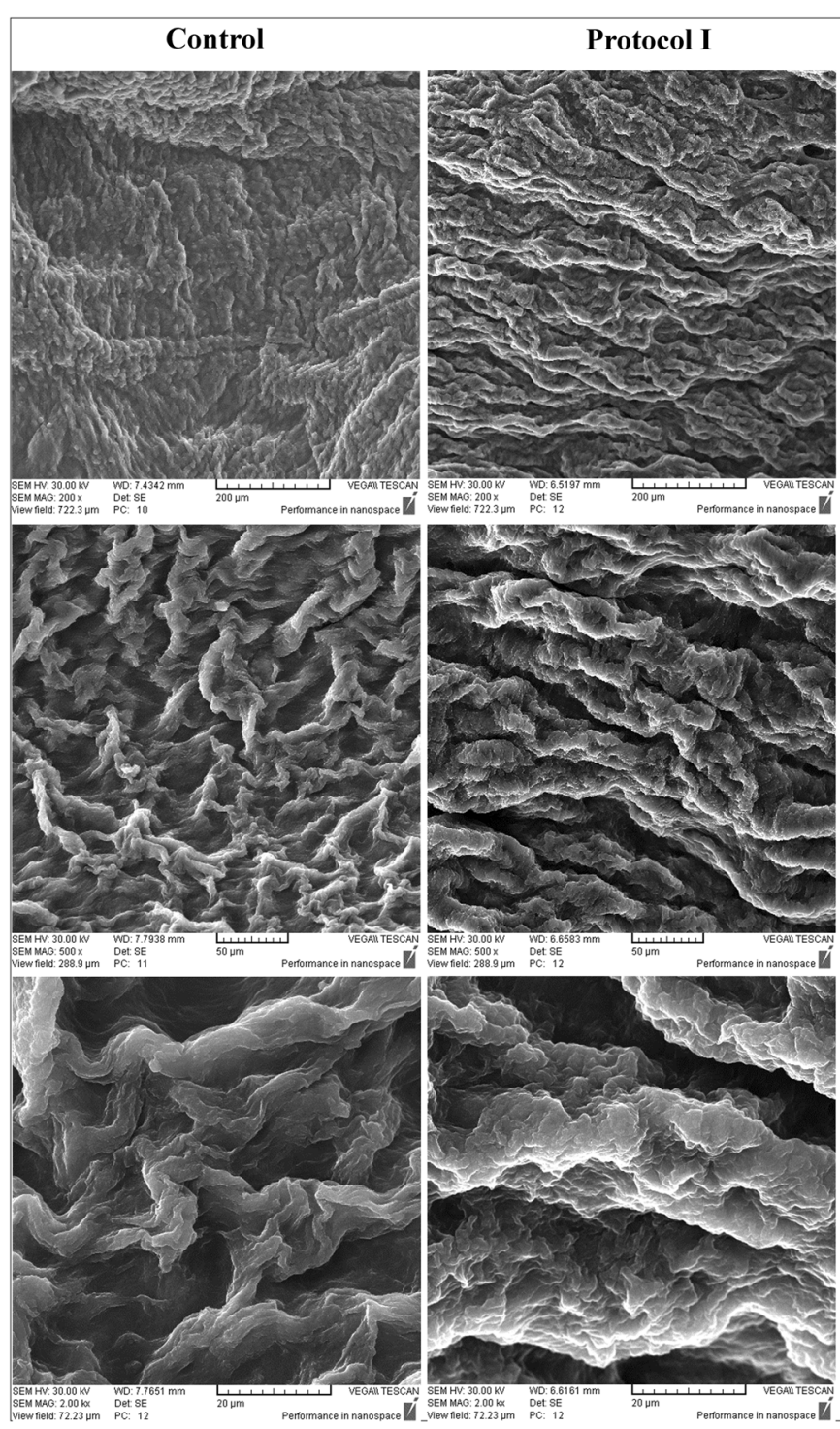

Fig. 4 SEM studies depicted that the replacement of the cobblestoneshaped elements with empty pores in acellular samples (provided from the body and the uterine horns) was indicative of complete decellularization in all protocols. Also, acellular samples showed no

evaluations of the specimens were suggestive of a regenerative process in the acellular tissue; endometrium and myometrium were detected in the grafted scaffolds, and a prominent vascularization process was observed in the implanted samples (Fig. 6).

\section{Discussion}

In this study, we decellularized ovine uteri by three different protocols and investigated the scaffolds' properties. This preliminary study introduces an optimized uterine decellularization method and paves the path for future studies on various utilizations of the scaffolds.
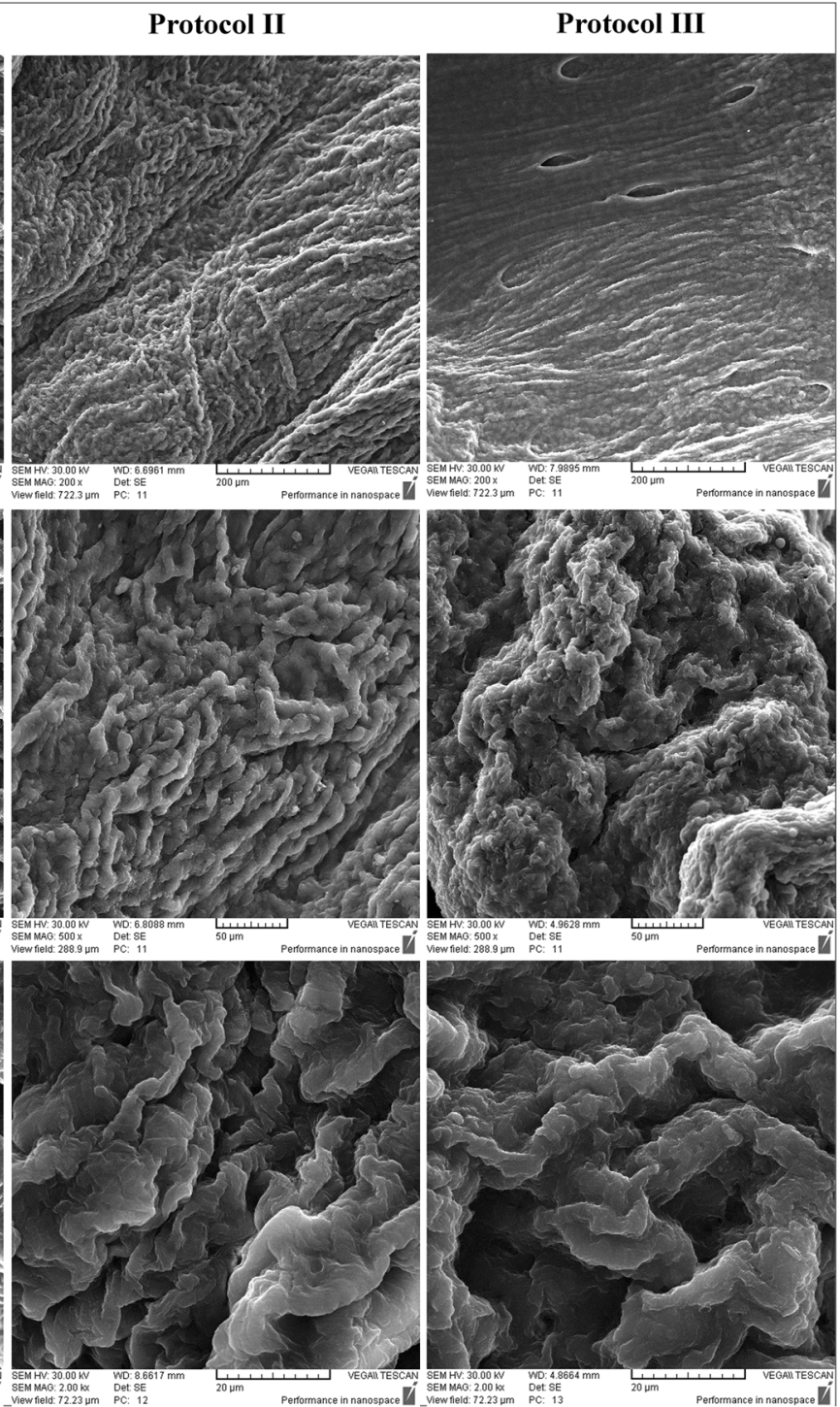

malformation or distortion comparing with the native tissue suggestive for the preservation of the ECM ultra-structure following the decellularization process

Application of acellular and tissue engineered natural scaffolds has already demonstrated a promising potential for substitution of various organs and regeneration of damaged tissues such as pancreas [16], arteries [17], and myocardium [18, 19]. Therefore, various investigations have been focused on the utilization of engineered tissues for regeneration of damages uteri or complete substitution of the organ by a nonimmunogenic uterus for AUFI patients $[6,20]$. Determination of an optimal decellularization protocol to generate an ideal natural scaffold is necessary for the possible clinical application of acellular tissues and organs. In 2014, Hellström et al. compared the efficacy of three perfusionbased protocols for decellularization of rat uterus and introduced two efficient methods to provide bioscaffolds with 
Fig. 5 CT angiography (a) assessment illustrates intact uterine large vessels in all specimens. Protocol III demonstrates the most affluent microvasculature compared with the other samples; protocol I and protocol II illustrate maintained large conduit network.

Biomechanical evaluation (b) of the samples ( $n$ 3; (A) native, (B) protocol I, (C) protocol II, (D) protocol III) indicates that the native tissue's Young's modulus is $0.8 \pm 0.5 \mathrm{MPa}$ which is significantly lower than that of protocols I $(4.5 \pm 2.4 \mathrm{MPa})$ and II $(3.2 \pm$ $1.7 \mathrm{MPa})(p<0.05)$. Young's modulus of protocol III (1.63 \pm $0.9 \mathrm{MPa}$ ) is not significantly different from the native tissue and shows the highest tensile strength comparing with the other scaffolds $(p>0.05)$
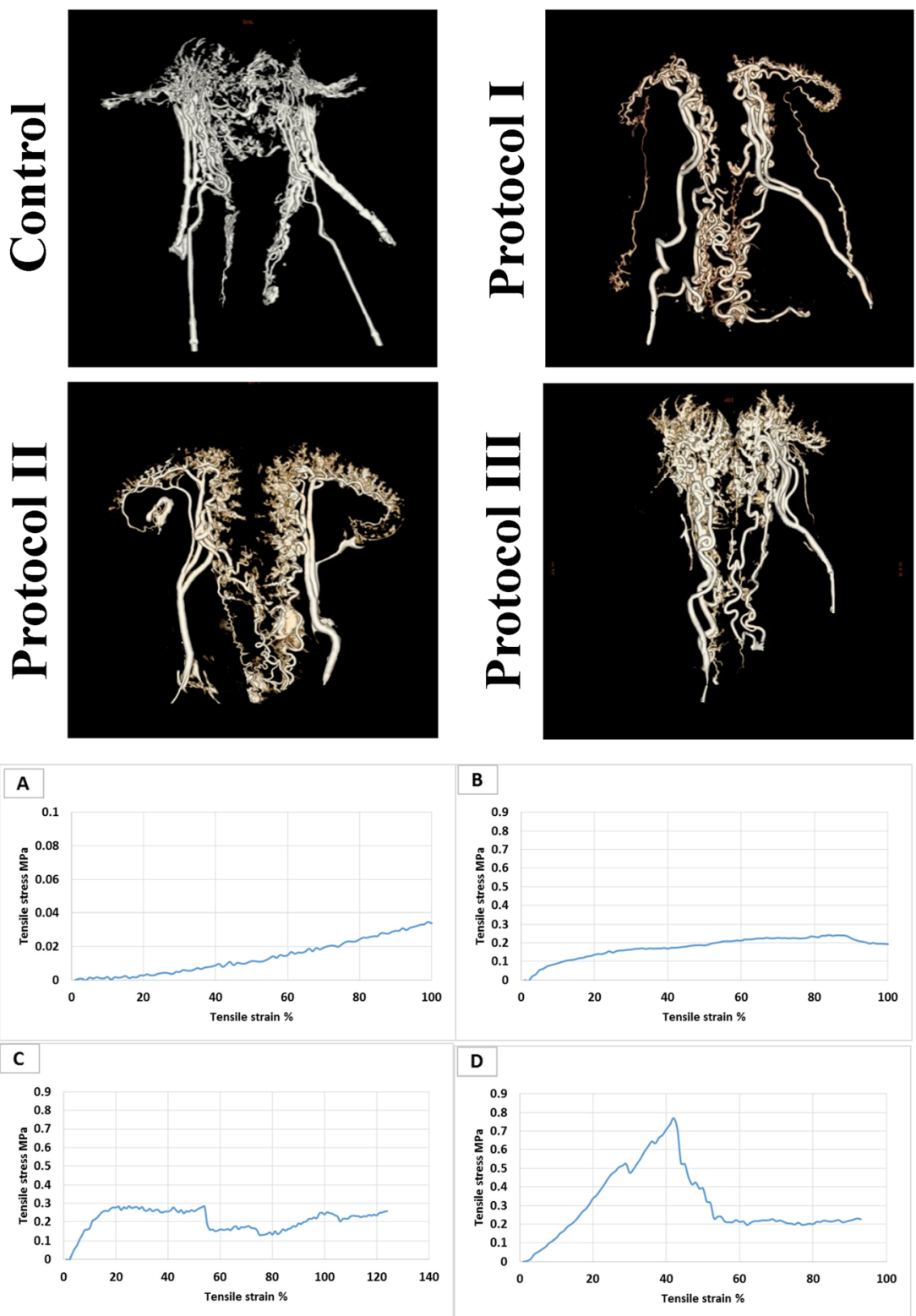

similar characteristics to the natural tissue [11]. Moreover, Miyazaki and Maruyama provided acellular rat uteri and investigated in vitro and in vivo recellularization capacity of the bioscaffolds; the results were encouraging regarding epithelial layer regeneration and angiogenesis [12]. Subsequently, Hellström et al. demonstrated the first pregnancy in acellular patch-implanted uterus of rat. The uteri were decellularized by two perfusion-based protocols, and $5 \times 20 \mathrm{~mm}$ patches were implanted on the host tissue; however, no pregnancies occurred on the grafted zone [13]. These studies provided the basis for further investigations on uterine tissue engineering in larger animal models.

Hereby, we compared the efficacy of three whole-organ decellularization protocols of the ovine uterus as a large animal model's organ. Preservation of the ECM and its protein fibers following the decellularization process is an imperative feature of an optimal protocol due to the considerable role of these proteins in the scaffolds' properties and functionality [21]. The outcomes of this study indicated that P2 (4\% DMSO and $1 \%$ Triton) and P3 (0.25\% SDS, 0.5\% SDS, and $10 \%$ neutral buffered formalin) scaffolds are associated with the highest amount of preserved ECM proteins which is probably attributed to the usage of a lower percentage SDS. The similarity of the ECM components to the native uterus tissue is beneficial for further application of the scaffolds since it provides a native-resembling environment for endometrial and myometrial cells.

The biomechanical characteristic similarities between protocol III scaffolds and the native uterine tissue could be related to the lower concentration of the detergents resulting in the 


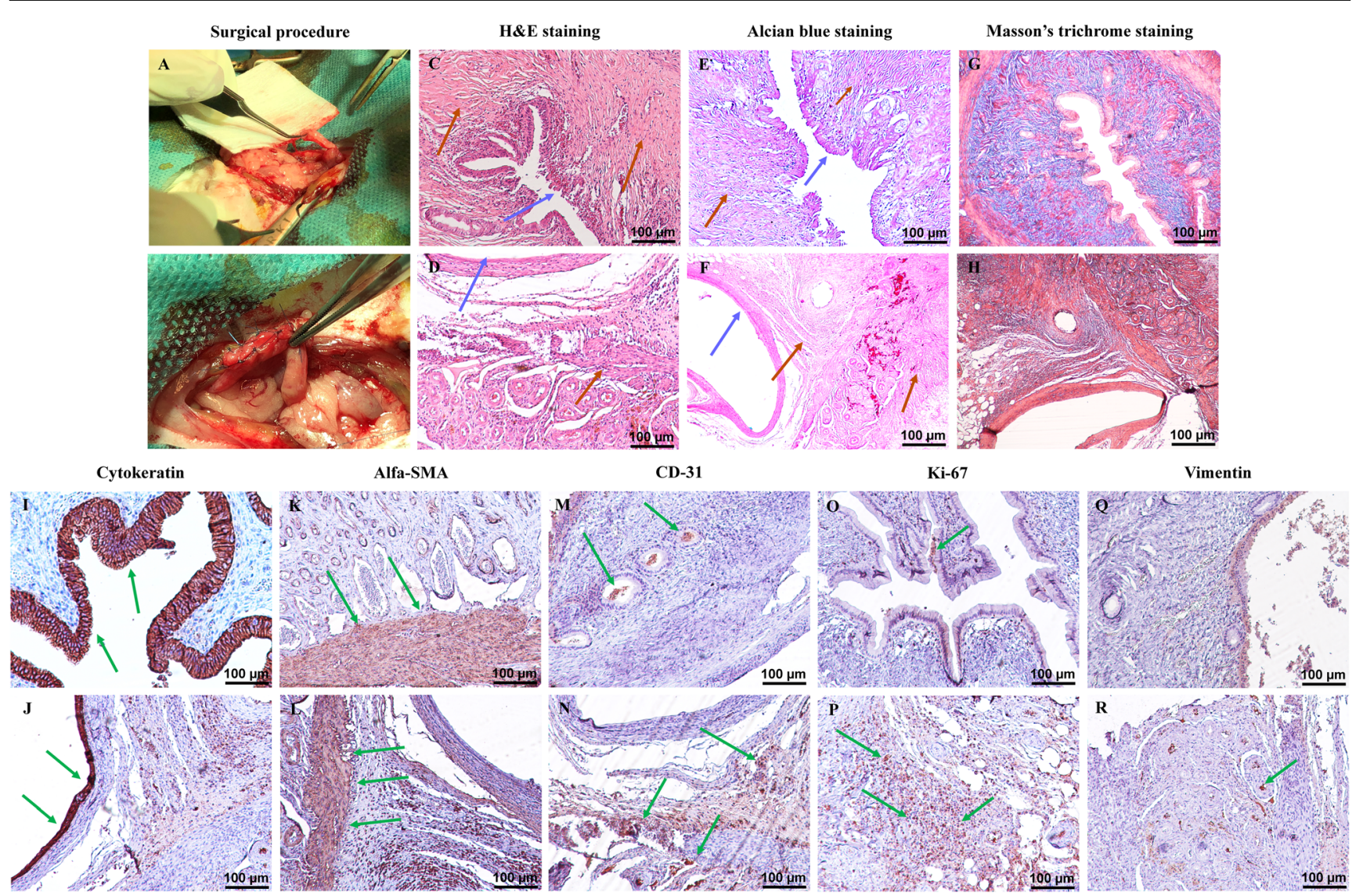

Fig. 6 Scaffold implantation and histological and immunohistochemical examination. The surgical procedure comprises (A and B) implantation of $10 \times 5 \mathrm{~mm}^{2}$ segments of protocol III scaffolds into the right uterine horns of eight female rats using interrupted 5-0 nylon sutures. H\&E staining of the native rat uterus (C) and the grafted scaffolds (D) shows the endothelial layer as the inner epithelial layer in both samples. The myometrium including smooth muscle fibers and collagen fibers is detectable in both samples (blue arrows indicate the endometrium, and brown arrows are used to illustrate the myometrium). Alcian blue staining of the control samples (E) and the implanted tissues (F) confirms the structural maintenance of the ECM and recellularization of the grafted samples (blue arrows indicate the endometrium, and brown arrows are used to illustrate the myometrium). Trichrome staining of the controls $(\mathrm{G})$ and the grafts $(\mathrm{H})$ illustrates muscle fibers with red color and the collagen fibers as blue fibers in all of the specimens which are relatively similar in structure and content. Immunohistochemical evaluation (positive-stained cells are in brown color; green arrows indicate stained cells) for cytokeratin marker in the control (I) and the implanted samples ( $\mathrm{J}$ ) shows the endometrium as intact inner epithelial layer in both groups. Alfa-SMA staining of natural rat uterus $(\mathrm{K})$ and implanted acellular samples $(\mathrm{L})$ demonstrates a considerable positive reaction in both groups which confirms the presence of smooth muscle cells in the myometrium. CD31 marker staining of the native $(\mathrm{M})$ and grafted $(\mathrm{N})$ tissues show diffused positive reaction in both groups. The grafted samples demonstrate increased positive-stained cells. IHC staining for Ki-67 in native tissue $(\mathrm{O})$ shows a weak positive reaction, and the implanted scaffolds $(\mathrm{P})$ a prominently higher positive reaction for Ki-67 marker both in the endometrium and the myometrium. IHC staining for vimentin illustrated the diffuse fibro-connective tissue in the myometrium of the native $(\mathrm{Q})$ and grafted $(\mathrm{R})$ specimens with no considerable difference

$\mathrm{CT}$ angiographic studies indicated that $\mathrm{P} 3$ was the most efficient protocol concerning the maintenance of the tissue's microvasculature. Thus, this decellularization protocol has an eminent potential to be exercised in further investigations on recellularization and application of uterine scaffolds. Maintaining the vascular network of an acellular organ to provide a transplantable scaffold with the capability of exchanging the essential molecules is a notable advantage of whole-organ decellularized scaffolds [24]. On the other hand, preservation of the conduits' network through the decellularization process is a prominent obstacle of this technique. Previous studies revealed that a principal challenge of bioscaffold production is the maintenance of the organ's lagenous products $[15,23]$. 
vascular network following the decellularization process [25, 26].

We performed cytotoxicity evaluations by MTT assay which determined P3 as the most viable scaffold, probably due to the lower concentration of SDS. These findings showed that this protocol merits further investigation of its potential for in vitro, in vivo, and clinical application. Previous studies illustrated that the remnants of detergent agents such as SDS and Triton have a notable cytotoxic effect which could hamper further in vitro and in vivo utilization of the acellular tissue [27], 28].

Overall, our evaluations distinguished P3 as an optimized decellularization protocol of the ovine uterus. Thus, we grafted $10 \times 5 \times 5 \mathrm{~mm}^{3}$ segments of these acellular tissues to the uterus of female rat models to illustrate the in vivo characteristics, biocompatibility, and toxicity of the scaffold as well as its potential for recellularization and uterine tissue regeneration. The outcomes were compatible with previous MTT assessments and confirmed the biocompatibility of protocol III scaffolds.

Histological and immunohistochemical examinations of the implanted scaffolds were suggestive for a regenerative process in the acellular tissues and recellularization in the endometrium and myometrium. Previous studies suggested that in vivo application of acellular tissues could result in recellularization of the bioscaffold which is probably pertaining to the host's circulating and in situ source of stem cells [16].

Moreover, a prominent angiogenesis process could be observed in the grafts which were considerably higher than the control samples. We investigated K-i67 marker staining as an indicator for proliferation which demonstrated a notably higher reaction in the grafted specimens. Altogether, our evaluations were suggestive for recellularization of the endometrium and myometrium of the scaffolds; the prominent angiogenic and proliferative capacity of the acellular tissues was also demonstrated.

Previously, Campo et al. decellularized the porcine uterus as a large model using SDS and Triton X-100. Although this pioneering study and the comparison between free-thawed and fresh tissues provided valuable data concerning the whole-organ decellularization of large animal uteri, the limited number of acellular organs ( $n$ 6) and the lack of comparison between the efficacies of different detergent agents could be considered as limitations of this study. Also, no data was reported regarding the biomechanical characterization of the generated scaffolds which is of eminent importance due to the physiologic role of the organ [14].

This study is hampered by some limitations. We aimed to develop an efficient decellularization protocol for a human-sized uterus and evaluate the bioscaffold biocompatibility and its recellularization potential. This study confirms uterine tissue regeneration after 10 days of follow-up; however, further investigations are required to confirm these findings after longer periods of time. The absence of hydroxyproline content assessment is a restriction of this investigation. In this preliminary study, the assessment of the grafted samples and the rat uterus was focused on the investigation of the regenerative potential of the bioscaffolds. Histological staining provided qualitative data on rat uterus and the grafted sample protein content. Subsequent studies could extend the findings in this rather novel field of research by evaluation of the protein content in the grafted and the host tissue. Western blot analysis of uterine biomarkers could provide valuable data regarding specific components of the scaffolds and grafts which is absent in this study; therefore, we suggest that future investigations should perform this technique for better evaluation of the bioscaffolds' properties. Although we obtained the ovine uteri from adult and fertile animals, the reproductive status, age, and the number of gravidity and parity of the donors, which could affect the tissue's properties, were undetermined.

In conclusion, this investigation compared three methods for decellularization of a human-sized uterus and introduced an optimized protocol to obtain bioscaffolds with native-like characteristics and a prominent potential for regeneration and angiogenesis. To the best of our knowledge, this is the first study to report biomechanical properties and angiographic evaluations of a large animal uterine scaffold. The introduced decellularization protocol could be employed in future in vitro and in vivo studies, and the outcomes of this study would bring us one step closer to the ultimate goal of whole human uterus bioengineering. We believe that further investigations in larger animal models for an extended period should be carried out to examine various aspects of the application of these bioscaffolds. Future studies could also be focused on whole-organ recellularization of the acellular scaffolds in a laboratory setting. Whole-organ bioengineering is still in its initial stages of development.

Acknowledgments We would like to express our sincere gratitude to Dr. Torabi for providing the organs and Mr. Reza Esmaili and Mr. Nourbakhsh for their kind cooperation during this project.

Funding This study was funded by Tehran University of Medical Sciences (grant number 96-03-30-36497).

\section{Compliance with ethical standards}

All the animal procedures were approved by The Animal Ethics Committee of the Tehran University of Medical Sciences, School of Medicine and Education Section of Basic Sciences and were performed in accordance with the Animal Welfare Act and the Guide for the Care and Use of Laboratory Animals.

Conflict of interest The authors declare that they have no conflict of interest. 


\section{References}

1. Milliez J. Uterine transplantation FIGO Committee for the ethical aspects of human reproduction and women's health. Int J Gynaecol Obstet. 2009;106:270.

2. Kisu I, Mihara M, Banno K, Umene K, Araki J, Hara H, et al. Risks for donors in uterus transplantation. Reprod Sci. 2013 Dec;20(12): 1406-15. https://doi.org/10.1177/1933719113493517.

3. Kisu I, Banno K, Mihara M, Suganuma N, Aoki D. Current status of uterus transplantation in primates and issues for clinical application. Fertil Steril. 2013;100(1):280-94.

4. Brännström M, Johannesson L, Bokström $H$, Kvarnström N, Mölne J, Dahm-Kähler P, et al. Live birth after uterus transplantation. Lancet. 2015;385:607-16.

5. Mats Brännström. Uterus transplantation and beyond. J Mater Sci Mater Med (2017) 28:70DOI https://doi.org/10.1007/s10856-0175872-0, 70 .

6. Hellström M, Bandstein S, Brännström M. Uterine tissue engineering and the future of uterus transplantation. Ann Biomed Eng. 2017 Jul;45(7):1718-30 Epub 2016 Dec 19.

7. Park DW, Choi DS, Ryu HS, Kwon HC, Joo H, Min CK. A welldefined in vitro three-dimensional culture of human endometrium and its applicability to endometrial cancer invasion. Cancer Lett. 2003;195:185-92.

8. Heidari Kani M, Chan EC, Young RC, Butler T, Smith R, Paul JW. 3D cell culturing and possibilities for myometrial tissue engineering. Ann Biomed Eng. 2017;45(7):1746-57.

9. Santoso EG, Yoshida K, Hirota Y, Aizawa M, Yoshino O, Kishida A, et al. Application of detergents or high hydrostatic pressure as decellularization processes in uterine tissues and their subsequent effects on in vivo uterine regeneration in murine models. PLoS One. 2014;9(7):e103201.

10. Hiraoka T, Hirota Y, Saito-Fujita T, Matsuo M, Egashira M, Matsumoto L, et al. STAT3 accelerates uterine epithelial regeneration in a mouse model of decellularized uterine matrix transplantation. JCI insight. 2016;1(8).

11. Hellström M, El-Akouri RR, Sihlbom C, Olsson BM, Lengqvist J, Backdahl $\mathrm{H}$, et al. Towards the development of a bioengineered uterus: comparison of different protocols for rat uterus decellularization. Acta Biomater. 2014;10(12):5034-42.

12. Miyazaki K, Maruyama T. Partial regeneration and reconstruction of the rat uterus through recellularization of a decellularized uterine matrix. Biomaterials. 2014;35(31):8791-800.

13. Hellström M, Moreno-Moya JM, Bandstein S, Bom E, Akouri RR, Miyazaki K, et al. Bioengineered uterine tissue supports pregnancy in a rat model. Fertil Steril. 2016;106(2):487-96 el.

14. Campo H, Baptista PM, Lopez-Perez N, Faus A, Cervello I, Simon C. De- and recellularization of the pig uterus: a bioengineering pilot study. Biol Reprod. 2017;96(1):34-45.

15. Barakat O, Abbasi S, Rodriguez G, Rios J, Wood RP, Ozaki C, et al. Use of decellularized porcine liver for engineering humanized liver organ. J Surg Res. 2012;173(1):e11-25.
16. Hashemi J, Pasalar P, Soleimani M, Khorramirouz R, Fendereski K, Enderami SE, et al. Application of a novel bioreactor for in vivo engineering of pancreas tissue. J Cell Physiol. 2018;233(5):380516.

17. Kajbafzadeh AM, Khorramirouz R, Kameli SM, Fendereski K, Daryabari SS, Tavangar SM, et al. Three-year efficacy and patency follow-up of decellularized human internal mammary artery as a novel vascular graft in animal models. J Thorac Cardiovasc Surg. 2019;157(4):1494-502.

18. Khorramirouz R, Kameli SM, Fendereski K, Daryabari SS, Kajbafzadeh AM. Evaluating the efficacy of tissue-engineered human amniotic membrane in the treatment of myocardial infarction. Regen Med. 2019;14(2):113-26.

19. Kitahara H, Yagi H, Tajima K, Okamoto K, Yoshitake A, Aeba R, et al. Heterotopic transplantation of a decellularized and recellularized whole porcine heart. Interact Cardiovasc Thorac Surg. 2016;22(5):571-9.

20. Young RC, Goloman G. Allo- and xeno-reassembly of human and rat myometrium from cells and scaffolds. Tissue Eng A. 2013;19(19-20):2112-9.

21. Ott HC, Matthiesen TS, Goh SK, Black LD, Kren SM, Netoff TI, et al. Perfusion-decellularized matrix: using nature's platform to engineer a bioartificial heart. Nat Med. 2008;14(2):213-21.

22. Myers KM, Elad D. Biomechanics of the human uterus. Wiley Interdiscip Rev Syst Biol Med. 2017;9(5).

23. Bhrany AD, Lien CJ, Beckstead BL, Futran ND, Muni NH, Giachelli CM, et al. Crosslinking of an oesophagus acellular matrix tissue scaffold. J Tissue Eng Regen Med. 2008;2(6):365-72.

24. Baptista PM, Siddiqui MM, Lozier G, Rodriguez SR, Atala A, Soker $\mathrm{S}$. The use of whole organ decellularization for the generation of a vascularized liver organoid. Hepatology (Baltimore, Md). 2011;53(2):604-17.

25. Williams C, Liao J, Joyce EM, Wang B, Leach JB, Sacks MS, et al. Altered structural and mechanical properties in decellularized rabbit carotid arteries. Acta Biomater. 2009;5(4):993-1005.

26. Arenas-Herrera JE, Ko IK, Atala A, Yoo JJ. Decellularization for whole organ bioengineering. Biomed Mater (Bristol, England). 2013;8(1):014106.

27. Cebotari S, Tudorache I, Jaekel T, Hilfiker A, Dorfman S, Ternes W, et al. Detergent decellularization of heart valves for tissue engineering: toxicological effects of residual detergents on human endothelial cells. Artif Organs. 2010;34(3):206-10.

Publisher's note Springer Nature remains neutral with regard to jurisdictional claims in published maps and institutional affiliations. 